\title{
Treatment of Class II div I malocclusion with quadri block-a modified fixed twin block appliance
}

\begin{abstract}
This case report demonstrates the use of modified twin block 'quadri block' appliance for treatment of 14 years old girl with Class II div 1 malocclusion having crowed maxillary and mandibular arches. After leveling and aligning of dental arches with nitinol wires patient was put on quadri block appliance for correction of molar relationship and convex profile. As the leveling of arches is carried out initially using fixed mechanotherapy; finishing is much faster than the conventional standard twin block appliance.
\end{abstract}

Keywords: modified fixed twin block, quadri appliance
Volume 9 Issue 2 - 2018

\author{
Arvind M,' Sujala Ganapati Durgekar,' Arun \\ Kumar $\mathrm{G}^{2}$ \\ 'Department of Orthodontics and Dentofacial Orthopaedic, \\ K.L.E. Society's Institute of Dental Sciences and Hospital, India \\ 2Department of Orthodontics and Dentofacial Orthopaedic. \\ Sharavati Institute of Dental Sciences, India
}

\begin{abstract}
Correspondence: Sujala Ganapati Durgekar, Reader Department of Orthodontics and Dentofacial Orthopaedic, K.L.E. society's Institute of Dental Sciences and Hospital, Yeshwanthpur Suburb, Bangalore-560022, Karnataka, India, Tel 9916623547,Email sujala2003@yahoo.com
\end{abstract}

Received: March 12, 2017 | Published: March 05, 2018

\section{Introduction}

In growing patients. Class II malocclusion can be corrected by various modalities. Among all methods, functional appliances bring about better facial esthetics and functional harmony. Twin block is a most common among all functional appliances used to the correct skeletal discrepancy in growing individuals. ${ }^{1-4} \mathrm{~A}$ crowded dental arches necessities an additional prefunctional orthodontics to align the dental arches for improvement of arch forms and to achieve a stable occlusion. Hence treatment of patients presenting with combination of crowding in arches and skeletal discrepancy requires increased treatment time; which further increases need for compliance from patients. In recent years, large number of fixed attachments has been developed to integrate functional mandibular advancement with fixed appliance therapy with each attachment having their own advantages and disadvantages..$^{5-8}$ Hence this article is an attempt to introduce a new modified fixed twin block design, which can be used in adjunct with fixed appliance. This modified fixed twin block helps us to correct the skeletal discrepancy optimally; as the functional phase is preceded by prefunctional fixed orthodontic appliance therapy to overcome the problem of pathologic construction bite in cases with crowded dental
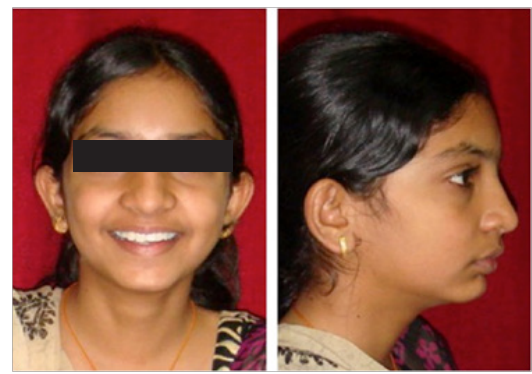

Figure I Pretreatment extraoral photographs showing convex profile. arches and with reduced over jet due to dental compensations. Hence, this design reduces treatment time and utilizes residual growth of patient efficiently to correct skeletal discrepancy.

\section{Case presentation}

A 14 years girl came to the department of orthodontics and dentofacial orthopaedics with a chief complaint of forwardly and irregularly placed maxillary anterior teeth. She was physically healthy and had no history of medical and dental trauma. No signs and symptoms of temporomandibular joint dysfunction were noted at the initial examination.

\section{Diagnosis}

The extraoral clinical examination showed a symmetric face with a convex profile caused by retrognathic mandible and deep mentolabial sulcus (Figure 1). Intraorally, there was half cusp Class II molar relationship with overjet of $6 \mathrm{~mm}$ and overbite of $4 \mathrm{~mm}$. There was moderate crowding of $6 \mathrm{~mm}$ in both maxillary and mandibular arches (Figure 2). Both the dental arches were symmetrical and $U$ shaped. The visual treatment objective (VTO) was positive.
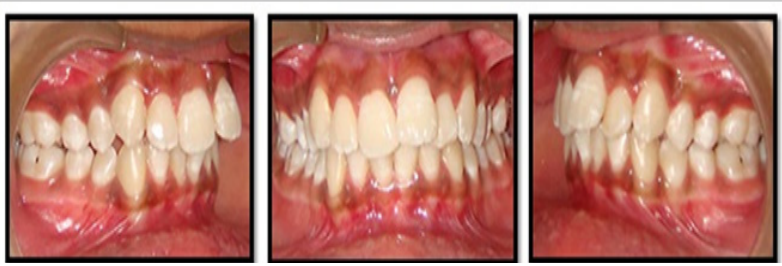

Figure 2 Pretreatment intraoral photographs showing crowded dental arches. 


\section{Treatment objectives:}

1. To level and align the maxillary and mandibular arches.

2. To achieve a Class I canine relation bilaterally

3. To achieve a Class I molar occlusion

4. Reduce the convexity of the profile.

\section{Treatment alternatives}

Three treatment options were considered .The first option involved extraction of upper first premolar and utilization of space for decrowding maxillary arch and proximal stripping in mandibular arch. This option would not correct the convex profile of the patient, which is due to mandibular retrognathism. The second option would be distalization of maxillary molar for correction of molar relationship as well as for decrowding maxillary arch. This option would resolve molar relation, but would worsen the profile of patient by clockwise rotation of mandible due to wedging effect caused by distal movement of molar. So third option of bite jumping with the help of myofunctional appliance was selected as it would not only correct the molar relation but also would also correct the underlying skeletal discrepancy and improve the overall facial esthetics.

\section{Treatment progress}

Overall treatment of patient the was divided into following stages:

Stage 1: Prefunctional orthodontics:

Based upon space analysis, the dental arches are decrowded by proximal stripping (Figure 3). Alignment and leveling of arches was carried out using 0.022 inch slot preadjusted edgewise appliance (MBT prescription, Gemini brackets, 3M Unitek) and sequential Niti arch wires.
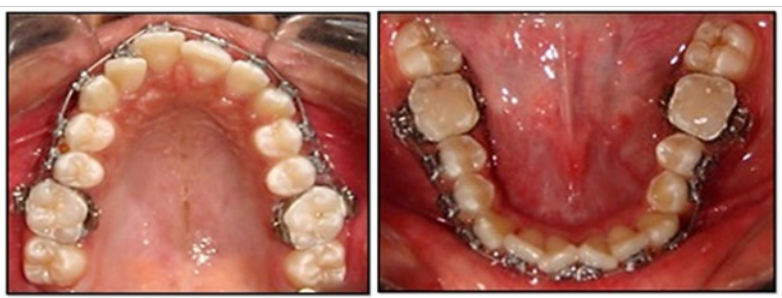

Figure 3 Aligning and leveling of dental arches in progress.

Stage 2: Consolidation of dental arches and construction bite:

After progressing to $21 \times 25$ stainless steel arch wire (Figure 4), maxillary and mandibular dental arches are consolidated and the construction bite is registered for fabrication of fixed twin block.

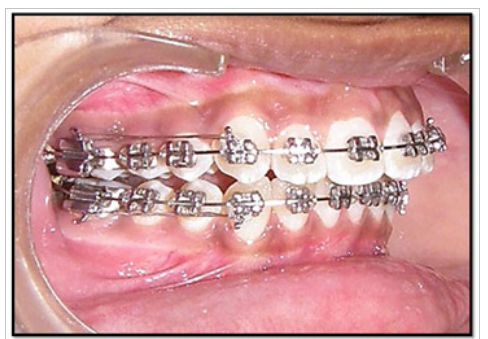

Figure 4 Dental arches stabilized with $21 \times 25$ stainless steel wire.
Stage 3: Construction of the appliance:

Components of appliance are as follows: (Figure 5) (Figure 6)

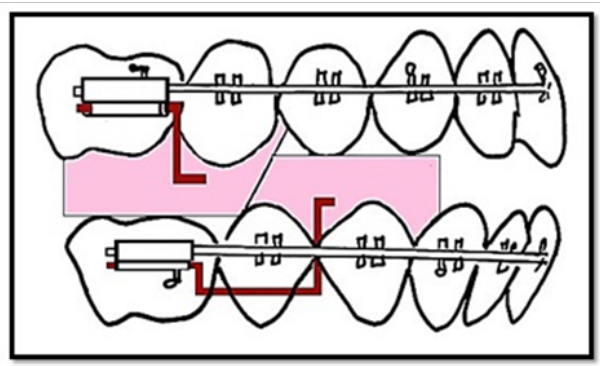

Figure 5 Maxillary and mandibular acrylic blocks inclined at 70 degree.
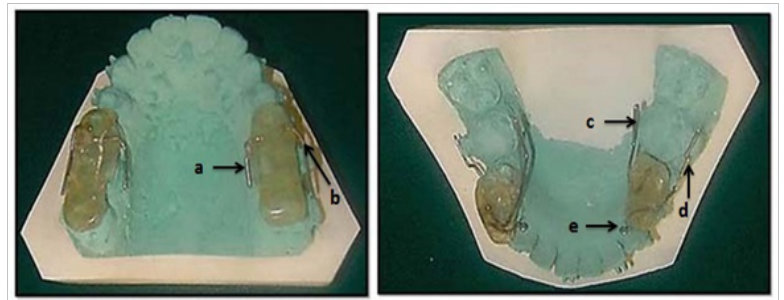

Figure 6 Maxillary and mandibular wire components. A) Palatal retentive tag. B) Buccal retentive tag. C) Lingual retentive tag. D) Buccal retentive tag. E) Ball end arm.

A new modified fixed twin block appliance has four blocks and each block is attached to buccal segments of stabilized dental arches. It works on same philosophy as of twin block appliance.

The appliance has acrylic and wire components.

1. Acrylic component

a. Maxillary right and left blocks.

b. Mandibular right and left blocks.

Maxillary and mandibular acrylic blocks are inclined at 70 degrees as in the standard twin block (Figure 5).

2. Wire components

A. Maxillary

i. Palatal retentive tag (made up of $0.7 \mathrm{~mm}$ stainless steel wire)

ii. Buccal retentive tag (made up of $1.00 \mathrm{~mm}$ stainless steel wire)

B. Mandibular

c. Lingual retentive tag

d. Buccal retentive tag

e. Ball end arm

All three above wire components are made of $0.7 \mathrm{~mm}$ stainless steel wire.

The buccal retentive tags are inserted in headgear tube for maxillary arch and accessory tube in mandibular arch respectively (both on first permanent molar). The palatal retentive tag and lingual 
retentive tag are inserted in lingual sheath on first permanent molar. The mandibular ball end arm is tied using the 0.009 ligature wire to lingual button bonded bilaterally on lingual aspect of mandibular canines. The working models are mounted on an articular with the construction bite in place (Figure 7). The construction bite is cut along the inclined planes from distal of maxillary first premolar to the distal of mandibular second premolar to create a wax pattern of acrylic blocks. The bend wire components are slightly heated and secured into the wax pattern (Figure 8). The maxillary and mandibular working models with construction bite and wire components are flasked and dewaxing of the block will be done. The wire components are safely secured in position by the investment materials. The heat cure acrylic is used to pack the flask and the appliance is constructed.

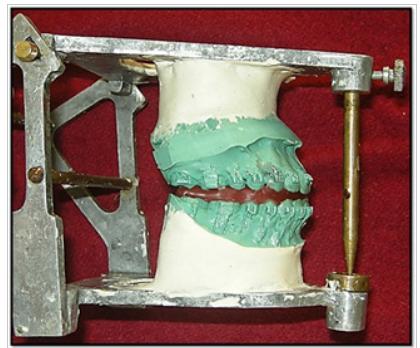

Figure 7 The working models mounted on an articular with a construction bite.
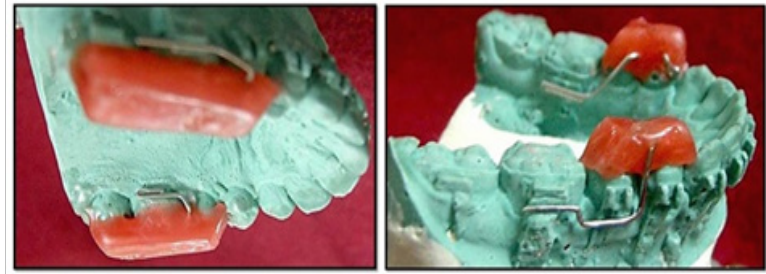

Figure 8 The wire components secured in a wax pattern.

Stage 4: Insertion of blocks:

$21 \times 25$ stainless steel arch wire is taken out from the brackets. The first molar bands are removed from the molars and the blocks are secured to the first molar bands using the wire components. The glass ionomer cement is applied to the first molar bands and the tooth facing surface of the acrylic blocks. The bands with the acrylic blocks are cemented to the buccal segment of teeth and the first molar (Figure 9).

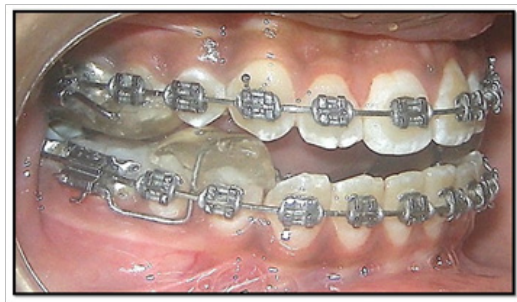

Figure 9 Intraoral photograph with quadri block appliance in place.

Stage 5: Myofunctional therapy:

This is the active myofunctional stage of the treatment, which extends till the desired mandibular advancement is achieved. There will be no trimming of acrylic blocks during this therapy as the curve of Spee is flattened during prefunctional orthodontics and contains continuous $21 \mathrm{X} 25 \mathrm{SS}$ wire in the lower arch.

\section{Stage 6: Post functional settling of occlusion}

After 6-7 months of twin block therapy, finishing and detailing of dental arches can be carried out. The 21X25 SS wire is cut distal to the mandibular canines and the posterior section of the wire is taken out of the lower buccal segment. The posterior vertical settling elastics are given for minor settling of the occlusion.

\section{Treatment results}

There was marked improvement in the facial esthetics (Figure 10) and smile of the patient. After appliance removal, an adequate relation was found between upper and lower teeth in all spatial planes with ideal overjet and overbite. Well interdigited buccal occlusion with Class I buccal occlusion was obtained (Figure 11).

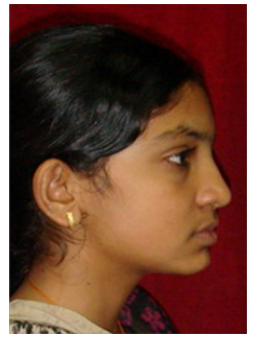

Figure 10 Post treatment extraoral photographs with pleasing profile
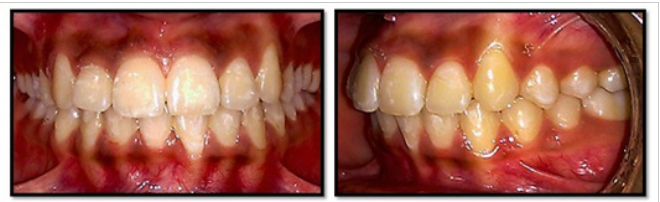

Figure I I Post treatment intraoral photograph with good Class I occlusion.

\section{Discussion}

This qudri block appliance can be used when growing and requires prefunctional fixed appliance therapy to correct dental compensations. Patients giving pathologic construction during bite registration and who are at the end of their pubertal growth spurt or at the completion at their peak growth.

These are many advantages of this appliance. Following are few of them:

1. As twin block is integrated with fixed appliance the correction of skeletal discrepancy can be carried out for patients with crowded dental arches.

2. The appliance is less bulky and the palatal and lingual acrylic plates are eliminated.

3. The problem of pathologic construction bite can be eliminated which removes the need for sequential advancement of mandible due to palatally placed anterior teeth or crowded teeth.

4. As the leveling of arches is carried out initially using fixed mechanotherapy; finishing is much faster than the conventional standard twin block. In conventional standard twin block there is posterior open bite created during active twin block therapy and 
hence demands an additional retentive phase to settle occlusion using upper inclined plane.

5. It can be used in non-compliant patients as it is fixed intraorally.

6. Cost effective and easy laboratory procedure.

\section{Acknowledgements}

None.

\section{Conflicts of interest}

None.

\section{References}

1. Lund DI, Sandler PJ. The effects of twin Blocks: a prospective controlled study. Am J Orthod Dentofacial Orthop. 1998;113(1):104-10.

2. Jamilian A, Show Katbakhsh R, Amiri SS. Treatment effects of the R-applaince and twin block in Class II div 1 malocclusion. Eur J Orthod. 2011;33(4):354-8.
3. Mahamad IK, Neela PK, Mascarenhas R, et al. A comparison of twin block and forsus (FRD) functional appalince- a cephalometric study. Int $J$ Orthod Milwaukee.2012;23(3):49-58.

4. Yaqoob O, Dibiase AT, Fleming PS. Use of the Clark twin block functional appliance with and without an upper labial bow: a randomized controlled trail. Angle Orthod. 2012;82(2):363-9.

5. Ruf S, Pancherz H. Dentoskeletal effects and facial profile changes in young adults treated with the Herbst appliance. Angle Orthod. 1999;69(3):239-246.

6. Heining N, Goz GR. Clinical application and effects of the forsus spring. A study of new Herbst hybrid. J Orofac Orthop. 2001;62(6):436-50.

7. Jones G, Buschang PH, Kim KB, et al. Class II non-extraction patients treated with the forsus fatigue resistant device versus intermaxillary elastics. Angle Orthod. 2008;78(2):332-338.

8. Frye L, Diedrich PR, Kinzinger GS. Class II treatment with fixed functional orthodontic appliances before and after different therapeutic effects. J Orofac Orthop. 2009;70(6):511-27. 